\title{
La planificación como proceso social
}

Manuel Herrera Gómez*

Antonio Trinidad Requena*

Actualmente la planificación no goza de buena reputación. Para muchos nos encontramos ante un conjunto de actividades -muy ligadas al dirigismo, al exceso de regulaciones abstractas, a la proliferación de comisiones, reuniones de "expertos", etc.emergido junto al intervencionismo de los Estados nacionales y destinado a morir con ellos. Para otros, la planificación -nacida como el intento de eliminar la elección individual en nombre de una conciencia superior-, tras haber producido innumerables tragedias, está destinada a la desaparición al igual que su matriz "tecno-aristocrática" (F. A. HAYEK, 1973). Incluso hay quienes consideran que la planificación es un anacronismo: en la actualidad, la velocidad de las tecnologías y la complejidad de las materias impiden cualquier intento de planificación.

Son muchas las razones que nos permiten aplicar a tales posiciones el calificativo de simplistas. Fácilmente se puede demostrar, en clave histórica, que los momentos más fuertes de la planificación pública de tipo dirigista -desde Walter Rathenau a Herbert Hoover, desde Rexford Tugwell al MITI japonés- generalmente han nacido de las alianzas entre los poderes públicos y los intereses empresariales y económicos (D. F. NobLE, 1977). Empíricamente se puede mostrar cómo el abandono de la planificación pública, lejos de conducir a un mayor bienestar colectivo, traslada a una rápida decadencia de las infraestructuras y del capital social y humano. Incluso se puede evidenciar que los sectores industriales más necesitados de innovación tecnológica registran el mayor uso de metodologías de previsión.

Oponer negación a negación, simplificación a simplificación nunca ha sido una estrategia intelectualmente adecuada. Es difícil negar que los críticos de la planificación en algunos casos tienen razón. Muchas de las actividades de planificación se han apoyado en una teoría inadecuada o simplista: con frecuencia los planificadores han cometido el error de pensar el poder decidir, en nombre de una conciencia superior, aquello que los ciudadanos podían hacer o podían desear; el sistema político, en más de una ocasión, ha utilizado la planificación como una actividad paternalista, supliendo por definición a una sociedad civil que era considerada incapaz de planeamientos autónomos o de racionalidad a largo periodo.

La actual defensa del significado y de la relevancia de las actividades de planificación sólo puede realizarse mediante un profundo replanteamiento de la función, el planteamiento y la narrativa de este conjunto de actividades. Es necesario identificar argumentos suficientemente analíticos y abstractos que permitan reelaborar críticamente las experiencias disponibles y el pensamiento reflejo acumulado sin agotarse en ellos. Y es preciso superar la visión de la planificación como pura técnica, para observarla como un proceso social en el que interactúan sistemáticamente diversas exigencias funcionales y diferentes coaliciones de actores.

\section{Definiciones de planificación}

En la literatura de las Ciencias Sociales es posible encontrar numerosas definiciones de planificación. Según A. EDDISON (1975: 23), la planificación es "un proceso de preparación del conjunto de las decisiones referentes a intervenciones a realizar en el futuro, encaminadas a alcanzar determinados objetivos con medios óptimos, con la disponibilidad de aprender del resultado tanto nuevas y posibles decisiones, como nuevos objetivos a conseguir". Para E. R. AleXANDER (1992b: 73), la planificación puede ser descri- 
ta como "una actividad social u organizativa intencional dirigida a desarrollar una estrategia óptima para acciones futuras que pretenden conseguir un conjunto de objetivos deseados o bien resolver nuevos problemas que surjan en contextos complejos. Tal estrategia debe presuponer la capacidad y la voluntad de destinar recursos y de actuar de tal manera que pueda ser implementada". Por su parte, P. HaLl (1980: 1) sostiene que la planificación es "el conjunto de procesos a que se comprometen los que disponen sobre las previsiones lógicas precedentes a la decisión. Forman parte de la planificación la definición del problema, su análisis, la definición de la amplitud y de los objetivos de la intervención prevista, la proyección de futuro, el planteamiento de alternativas, su valoración, los procesos de decisión en la implementación, en la dirección, en el control y en la actualización". Teniendo presente el precio que conlleva toda síntesis, J. Forester (1989: 3) define la planificación como "la guia de una acción orientada al futuro" y J. FRIEDMAN (1967: 267) como "un proceso de guía encaminado al cambio interno de un sistema social".

Este breve recorrido nos permite revelar algunas coordenadas de referencia de la planificación como actividad especializada:

- La planificación es un proceso orientado al futuro. Todo intento de planificar requiere (aunque no se agota en esto) el esfuerzo de prever los posibles resultados de una intervención. En algunos casos, tal previsión podrá ser paramétrica, es decir, encaminada a individuar los cambios introducidos por la acción en un contexto considerado -excepto para aquella intervención- invariante. Sin embargo, en la mayoría de los casos tal previsión deberá ser estratégica: incluso asumirá como objeto de la previsión la gama de posibles reacciones de los actores presentes en el contexto y los posibles impactos concurrentes de otras decisiones emitidas desde el exterior.

- La planificación es un proceso con una alta intensidad de conocimiento. La planificación es una actividad práctica que extrae del conocimiento y de la técnica tanto la propia condición de posibilidad (capacidad de previsión), como su legitimación social y organizativa ${ }^{1}$. Los análisis y las propuestas del planificador son -0 aspiran a ser- producidas de forma intersubjetivamente vinculante. Sin embargo, respecto a las actividades científicas, la planificación se diferencia por ser, en última instancia, una actividad prescrita. La utilización del conocimiento científco por parte de un planificador no está orientada a incrementar el patrimonio de conocimiento disponible, sino a utilizar tales conocimientos con el fin de introducir cambios en el contexto respecto a direcciones pre-determinadas. Si esto no quiere decir que la actividad de planificación esté privada de una fuerte dimensión reflexiva (A. SCHON, 1983), es necesario reconocer que son radicalmente diferentes las formas de utilización del conoci- miento en la comunidad científica y en el proceso de planificación (M. S. FELDMAN, 1989).

- Tal conocimiento es un apoyo al proceso de decisiones, no a todo el proceso. La formulación de un análisis de las previsiones no elimina la necesidad de escoger: los objetivos deben seleccionarse a partir de un conjunto más bien amplio, y-dada la incertidumbre respecto al futuro y a la doble contingencia de las interacciones sociales- todo proceso de planificación identifica conjuntos de alternativas que posteriormente no pueden reducirse a partir de consideraciones exclusivamente técnicas (y análogas observaciones valen para la elección entre diversas modalidades de ubicación de los costes y de los recursos). La dimensión de la decisión -en otras palabras, la dimensión política- jamás puede cambiarse 0 anularse mediante un complejo de actividades técnicas y de elaboración. Esto permite distinguir la planificación -como conjunto de prácticas de apoyo a la decisión- de la tecnocracia -el intento de sustituir o "cancelar" al que decide-.

Por otra parte, del análisis de estas definiciones es posible derivar una visión de la planificación como una actividad de tipo (al menos en el caso ideal) cíclico, descomponible en fases de observación, decisión, implementación y evaluación. Todo proceso de planificación tiene su origen en la observación de un cambio percibido como problemático. Por observación se entiende en este contexto un conjunto de actividades que va desde el desarrollo de una "narrativa" -que atribuye un significado a tal fenómeno desde el punto de vista del contexto de referencia, articula la relevancia e individúa las "responsabilidades"- la recogida -más o menos disciplinada- de informaciones, a la articulación de previsiones sobre la evolución y sobre el impacto ${ }^{2}$. Es importante señalar que tal fase incluso puede comprender -como generalmente es el caso- un proceso de evaluación de las intervenciones precedentes o similares, en el interior de la misma organización o de otras. El tipo de observación que estamos tratando no tiene, sin embargo, finalidades contemplativas: aspira -ideal o fácticamente- a contribuir a estructurar una decisión relevante desde el perfil de la acción relativa a tales cambios ${ }^{3}$. Tal decisión da vida a procesos de implementación que pueden ir desde el cambio en las rutinas organizativas presentes en el contexto, hasta el diseño de nuevas estructuras organizativas ${ }^{4}$. En fin, la conclusión o estabilización de toda intervención requiere una evaluación respecto a los cambios en el ambiente y respecto a los objetivos que se quieran alcanzar ${ }^{5}$. Esta evaluación se enlaza idealmente a la primera fase o puede ser contemplada como el injerto de un nuevo ciclo de actividades planificadoras.

En este artículo el ciclo completo anteriormente descrito será denominado con el término "proceso de planificación". Sin embargo, como se verá más adelante, adoptar tal concepción extensiva implica una superación -y una crítica- de muchas defi- 
niciones consolidadas de planificación, definiciones que la reducen a operaciones de observación y evaluación. Según estas concepciones -que podemos denominar "clásicas"-, observación y evaluación son fases de la "planificación" en cuanto que están caracterizadas por una mayor intensidad de elaboración "objetiva”, por un mayor recurso a las competencias profesionales de los "planificadores" -entendidos como grupo profesional- y por una menor "confusión" entre previsión racional y consideraciones "políticas". Por su parte, las fases de decisión e implementación más bien serían dominio del que decide y de las autoridades internas de la organización. Sin embargo, a lo largo de estas páginas se sostendrá que el recurso al análisis de previsión y al conocimiento técnico no solamente se limita a la fase de observación, sino que se distribuye en todas las fases, y con particular intensidad en la fase de implementación (C. V. PATtOn, 1986). Igualmente distribuidas, incluso en la fase de observación, resultan las tensiones entre conocimiento técnico y selectividad organizativa y política (D. A. RocheForT, R. W. CoBb, 1994). La distinción entre planificación y decisión -y la división del trabajo entre los diversos roles- debe realizarse interactivamente en el interior de todas las fases, no a través de la separación entre las mismas fases (G. BENVENIST, 1991).

\section{Planificación individual $y$ planificación social}

En las definiciones anteriormente apuntadas, la planificación es presentada como un instrumento de racionalidad, entendida weberianamente en un doble sentido: por una parte, de incremento de la capacidad de previsión del resultado de los diversos cursos de acción y, por otra, de incremento de la calculabilidad de las ventajas y desventajas asociadas a cada uno de estos ${ }^{6}$. En otras palabras, toda forma de planificación es un intento de reducir la incertidumbre de las decisiones y de las operaciones. Llegados a este punto es necesario comprender por qué, frecuentemente, el proceso de planificación presenta un carácter conflictivo, y por qué la legitimidad misma de la elección de planificar se encuentra ante verdaderas oposiciones. Estos problemas requieren establecer una clara distinción entre la planificación como atributo de las acciones individuales y la planificación como proceso referido a un contexto social.

Desde el punto de vista de todo singular actor individual, la planificación es una precondición dada por descontada de la racionalidad de las propias acciones. Según la clásica definición parsonsiana, "una acción es siempre un proceso temporal (...). El concepto de fin de una acción implica siempre una referencia al futuro, a un estado que o no existe aún -y no existirá sin una intervención del actor- o que, en el caso de que ya exista, no permanecerá como tal sin su intervención" (T. PARSONS, 1937: 45). En esta orientación al futuro está implícito que el actor ejercite su capacidad de anticipar los cambios venideros del contexto y los compare con aquellos previstos en el caso de su acción. El carácter "racional" de una acción, la elección del curso de acción más eficiente, se basa en esta capacidad de anticipación. Toda acción racional es, o por lo menos aspira a ser, una acción correctamente planificada. Y, cotidianamente, las acciones que activamos requieren previsiones bastante complejas: por ejemplo, un individuo que quiera adquirir una calculadora normalmente afrontará problemas tales como la previsión de la innovación tecnológica (aquel modelo, ¿̨no se quedará anticuado muy pronto?), la evolución de las propias exigencias futuras y el comportamiento de otros actores (¿cuáles son los standards que utilizan las personas cuando intercambian datos?). Estas previsiones -desarrolladas incluso recogiendo informaciones específicas- deberán ligarse con las opciones y los recursos disponibles en el itinerario del proceso selectivo. A nivel individual, la elección no está entre planificar o no planificar, sino entre los diversos tipos de planificación y el problema de la escasez de planificación, no su exceso. Quien adquiere una calculadora -por ejemplo, a partir del color de la funda y entrando en el primer negocio que encuentrageneralmente no es considerado un modelo a imitar.

Sin embargo, el problema de planificar o no planificar se plantea con fuerza si el contexto de referencia es un sistema social y no un individuo. En este caso, la planificación no es un instrumento de apoyo a una decisión individual, sino una modalidad específica de gestión de la interdependencia entre más actores analíticamente independientes ${ }^{7}$. La planificación de un sistema social asume inevitablemente el carácter de un vínculo de las opciones de los actores, mediante una previsión centralizada y asimétrica de los comportamientos admisibles -en relación a un determinado objetivo-, de la ubicación secuencial de los comportamientos de las unidades y de las modalidades de acceso a los recursos y a las sanciones objeto de la planificación (J. D. Thompson, 1967).

Aquí las palabras clave son centralizada y asimétrica. Toda planificación referida a un contexto social -ya sea una organización, un área territorial, una asociación deportiva- es centralizada (respecto al nivel teóricamente posible de los intercambios entre los actores participantes) y asimétrica (no confía sólo en el consenso de las unidades participantes, sino modifica, de forma más o menos acentuada, el contexto de decisiones mediante decisiones jerárquicas). Por ejemplo, la existencia de un plan de ordenación urbana -una de las formas más comunes de planificación- determina precedentemente cualquier decisión individual sobre las posibilidades de utilización de un terreno, el tipo de operaciones que pueden realizarse y su valor de mercado; la decisión de quien aprueba el plan vincula al ciudadano que quiera 
construir. La planificación estratégica de una empresa determina las orientaciones y los sistemas de incentivos de las singulares divisiones y de los singulares repartos.

Por consiguiente, proponer una intervención de planificación, respecto a cualquier sistema social, implica asumir -y en los Estados democráticos argumentar- que es preferible sustraer de la interacción descentralizada entre los actores algunas dimensiones o ámbitos de decisión, sustituyéndolos con formas de coordinación jerárquica. Es comprensible que la perspectiva de planificar a nivel colectivo no se dé jamás por descontada, estando siempre disponible en tal nivel la alternativa constituida por el abstenerse de hacerlo. En otras palabras, la racionalidad del plan siempre encuentra una alternativa disponible en la racionalidad del mercado (DAHRENDORFF, 1966) ${ }^{8}$.

La relación planificación/mercado es uno de los problemas centrales de la teoría de la planificación. El mercado, analíticamente entendido, es un mecanismo de coordinación horizontal, operante entre las contrataciones descentralizadas de una amplia gama de actores independientes, cada uno dotado del mejor (en cuanto directo) conocimiento de los costes y de las oportunidades disponibles. Esta modalidad de coordinación permite una notable libertad de elección por parte de los actores y produce una importante dinámica social: la coordinación de mercado, integrada mediante el sistema de precios, es particularmente eficiente al trasmitir recíprocamente la información sobre las exigencias y los deseos de los actores, y es particularmente flexible para adaptarse a situaciones de incertidumbre y de cambio. Estas características y este nivel de flexibilidad no pueden ser producidas - por lo menos en la misma medida- por formas de coordinación jerárquica: la sustitución de la planificación centralizada por la coordinación de mercado tiene como resultado formas sociales inestables e ineficientes, además de condenadas -como han demostrado las economías planificadas- a recurrir a fuertes dosis de autoritarismo político. Si se pudiese pensar en subsumir el conjunto de las interdependencias sociales en el interior de los esquemas de mercado, razonablemente se debería concluir que los espacios de la planificación social están y son drásticamente limitados. Se debería asumir que el mercado, eventualmente integrado por la visión del sistema político como un mercado sui generis, es suficiente para garantizar niveles adecuados de coordinación y de gestión de las interdependencias y resultados más positivos para el sistema social en su conjunto, respecto a cualquier solución basada en la planificación intencional (C. LINDBLOM, 1977). Sin embargo, existen muchos elementos que indican la dificultad -por no decir la imposibilidad analítica- de reconducir las modalidades de coordinación social solamente a los intercambios de mercado. Y es propiamente de la exploración de tales situaciones de donde se puede derivar una base analítica para la teoría de la planificación capaz de integrar una visión -igualmente crucialde los límites de lo planificable.

\section{3. ¿Por qué planificar? Desde problema de los bienes públicos a la teoría de los costes de transacción}

La teoría clásica de la planificación democrática siempre ha reconocido, aunque con diferente intensidad, la importancia del mercado. Por tanto, no debe sorprendernos que la teoría democrática "clásica" de la planificación haya afrontado el problema del "por qué y cuándo planificar" en relación -y no en oposición- a la existencia del mercado, elaborando una respuesta en términos residuales: la intervención pública -y la planificación identificada con ella- se justifica en los casos en que la coordinación de mercado fracasa (J. FRIEDMAN, 1987). El planteamiento clásico, dominante durante todo el periodo post-bélico, se ha identificado estrechamente con la teoría de los bienes públicos (y/o colectivos), procediendo a través de dos fases:

a) Por una parte, asumiendo que la presencia de tales bienes justifica la intervención pública

b) Por otra, que la planificación está legitimada como el principal instrumento de racionalidad de esta última.

Los bienes públicos pueden definirse como aquellos bienes (o servicios) que todos los actores consideran indispensables e importantes, pero que no se consiguen producir de forma eficiente a través de los mecanismos del mercado. En sentido estricto, tales bienes están constituidos por todos los bienes y servicios privados de valores de mercado en cuanto que resulta imposible excluir a eventuales "no contribuidores" de su goce: bienes ambientales como la calidad del aire o del agua (u otros elementos naturales), bienes sociales como la defensa nacional o el mantenimiento del orden público, bienes culturales como la riqueza o el refinamiento de una lengua. Al no ser posible integrar tales bienes en el sistema de precios, una ubicación de mercado de estos bienes sería o completamente arbitraria o bien, asumiendo su valor como nulo, trasladaría a una excesiva explotación y, en consecuencia, a su rápida degradación y deterioro (G. HARDIN, 1968). Respecto a esta categoría de bienes, una intervención pública también es justificable en una economía de mercado.

Una segunda categoría de bienes (o servicios) que a menudo es considerada comparable -en términos de "fracasos del mercado"- está constituida por aquellos bienes que -en ausencia de cualquier regulación- serían ubicados en el mercado de forma ineficiente: es el caso de los bienes para los que existe un monopolio natural, por ejemplo la elaboración de electricidad o de los servicios en que existe una fuerte asimetría natural, que hace 
imposible al "cliente" valorar efectivamente la calidad del servicio recibido, como las prestaciones escolares o sanitarias. En fin, existen bienes o servicios, los llamados merit goods, que son considerados centrales para la identidad misma de la sociedad y, en consecuencia, son sustraídos del mercado en función del valor social atribuido (por ejemplo, la educación superior, museos, uso de transporte colectivo) $)^{9}$.

La intervención pública en una economía de mercado está justificada, según este planteamiento, por la existencia de estas categorías de bienes. Sin embargo, tal intervención debe resolver un problema de no poca relevancia: ¿cómo gestionar la trasmisión y evaluación de la información en ausencia de un sistema de precios? La coordinación de mercado -los procesos de ajuste entre los actores interesados en la consecución de objetivos privados- tiene lugar, por así decir, "automáticamente"10 gracias a las informaciones circuladas por el sistema de precios: el empresario que decida ignorar las informaciones sobre las preferencias de los consumidores, sobre su disponibilidad a pagar o sobre los procedimientos técnicos más eficientes para la producción de un bien, está obligado automáticamente a pagar los costes; quien realiza elecciones correctas está igualmente informado del resultado de las propias decisiones mediante el aumento de los retornos. En el caso de los bienes colectivos gestionados por un operador público, especialmente cuando éstos actúan en régimen de monopolio, tales mecanismos están, sin embargo, ausentes: derivan de riesgos constantes de sobreproducción, de escasa atención a los costes, de insensibilidad a las variaciones de la demanda y de colisión con los segmentos de productores o con las categorías de consumidores particularmente fuertes.

La necesidad de una alternativa al mercado para determinadas categorías de bienes, unida a la conciencia de la ausencia de mecanismos selectivos naturales, permite justificar, según la teoría clásica de la planificación, la racionalidad del plan. Los instrumentos de planificación son contemplados como un posible equivalente funcional del sistema de precios en el caso de la intervención pública: a la planificación es atribuido el objetivo de recoger sistemáticamente informaciones sobre la evolución de la demanda y de la oferta (y de garantizar la correspondencia futura mediante la previsión), "obligando" a los organismos públicos a la eficiencia. Una finalidad adicional, pero no olvidada, está constituida por la posibilidad, mediante la planificación, de articular las exigencias sociales de forma intersubjetivamente vinculante, reduciendo la tendencia del sistema político a privilegiar los intereses organizativos más influyentes. La planificación es asumida al mismo tiempo como una forma de tutela de los fracasos del mercado económico y de los fracasos del mercado político (P. Hall, 1980).

Este planteamiento del problema, de los ámbitos y de las funciones de la planificación está seguramente dotado de una notable elegancia y claridad conceptual. Por otra parte, tiene el mérito de contribuir a definir la alternativa público-privado en términos de una división del trabajo, más que no de oposición recíproca.

Sin embargo, la adopción de este cuadro conceptual presenta -en el caso de la teoría de la planificación- algunas dificultades que no deben olvidarse. En primer lugar, la coordinación jerárquica y la coordinación descentralizada son objetivadas en formas empíricas (Estado o Mercado), haciendo latente tanto la relevancia de la coordinación jerárquica en las actividades privadas, como la existencia de fenómenos de contratación descentralizada en el sector público. La identidad de la planificación, por consiguiente, es atribuida a la esfera de los actores públicos, terminando por ignorar o marginar la fuerte presencia y relevancia de los procesos de planificación en el sector privado o non pro$f_{i t}{ }^{11}$. En segundo lugar, al definir tanto la intervención pública como la actividad de planificación aludiendo a categorías de bienes y servicios, la función de la planificación es contemplada en términos de una capacidad técnica para identificar a priori el mejor modo de producir, distribuir o defender el bien mismo a través de la utilización de un conocimiento "superior" y más "objetivo". Esto ha tenido dos importantes consecuencias: respecto al sistema político, la planificación ha terminado generalmente por aspirar a asumir el rol de "consejera del príncipe", exonerándose de la confrontación con los intereses; respecto al aparato organizativo, los planificadores generalmente han restringido la propia actividad al momento de la formulación de las políticas, mientras la implementación de tales propuestas era considerada como efecto mecánico o como responsabilidad de un tercer actor ${ }^{12}$. En efecto, considerando al Estado como principal punto de referencia para el problema de los bienes públicos -y considerándolo, en cuanto caracterizado por algunas peculiaridades cruciales tales como el monopolio del uso legitimado de la fuerza física, "superior" respecto a otras estructuras sociales-, la teoría de la planificación ha terminado por asumir a los actores presentes en el ambiente como presencias paramétricas o como recipientes pasivos.

En los últimos veinte años, como respuesta tanto a los problemas empíricos como a las innovaciones conceptuales originadas en las Ciencias Sociales, ha emergido en la teoría de la planificación una nueva y más compleja perspectiva que trata de superar la dicotomía público-privado, ligando estrechamente la planificación a las formas de coordinación jerárquica entendidas analíticamente (E. R. AlEXANDER, 1992a, 1994; J. M. BRYSON y P. S. Ring, 1990; J. A. Robins, 1987). Según la declaración programática de E. R. ALEXANDER (1992a: 191), para esta perspectiva "la planificación no es complementar al mercado, mas bien es una propiedad de las organizaciones y de los sistemas interorganizativos en la medida en que tienden a ser jerárquicos, independientemente de que sean públicos o privados, o con caracteristicas mixtas".

Esta nueva perspectiva se avala, como cuadro de referencia teórico, del esquema teórico elaborado en los últimos veinte años 
por aquella corriente de las Ciencias Sociales, a caballo entre economía y sociología, definida como economía neo-institucionalista, economía no ortodoxa 0 -más recientemente- economía de los costes de transacción (O. E. WilliamsON, 1975; 1985; 1994). ¿Qué diferencia a esta perspectiva de la teoría económica ortodoxa? ¿Y por qué resulta tan apetecible para la teoría de la planificación?

El interés por los costes de transacción nace del intento de responder a la siguiente pregunta: :por qué en una economía de mercado existen numerosas realidades en las que la organización jerárquica que produce internamente es preferida a la posibilidad de combinar bienes, servicios y competencias externas adquiridas externamente, sobre el mercado? ¿Por qué los actores prefieren vincularse jerárquicamente más que horizontalmente a través de intercambios de mercado?

Para responder a este tipo de interrogantes, las premisas principales de la economía ortodoxa han sido revisadas:

- Ha sido necesario sustituir la asunción de la racionalidad perfecta -es decir, de capacidades computacionales para poder calcular la maximización de la propia función de utilidad- y de la completa información por el reconocimiento de la existencia de límites computacionales y la presencia de áreas de incertidumbre informativa que permiten a los actores estrategias oportunistas.

- Ha sido preciso remplazar la asunción de que los intercambios de mercado son standalizables, fluidos y reversibles por la hipótesis de un continumm de costes de transacción $^{13}$.

- Ha sido conveniente sustituir la asunción del individuo calculador como unidad de análisis por la transacción - unidad relacional- cuya dimensión crítica principal, la especificidad de la inversión solicitada, puede ser formulada en términos de dependencia bilateral.

Estos cambios han hecho posible la formulación de la tesis principal: la clave para la comprensión de la estructura socio-económica está constituida por diversas modalidades de resolución de los problemas derivados de las exigencias del gobierno complejo de estas transacciones (O. E. WilliamsOn, 1975).

Este cambio de perspectiva permite distinguir entre coordinación de mercado y coordinación jerárquica no en términos de categorías de bienes (privados/colectivos) o de estructuras jurídicas formales (empresas privadas/burocracia pública), sino en términos de características de interacción y organizativas de la producción y de la distribución. En algunos casos, los costes de transacción son nulos o bajos, los contactos episódicos y la reversibilidad constituye una opción fácilmente alcanzable por todos los patners que interactúan. En estos casos, parece difícil que las formas de control jerárquico puedan difundirse y -cuando ello suceda- estarán constantemente desafiadas por la negociación descentralizada. En otros casos, sin embargo, los costes de transacción representarán un elemento relevante: la interacción podrá requerir elevadas inversiones en elementos específicos difícilmente transportables fuera de aquel contexto -incrementando la propia vulnerabilidad respecto a una posible defección de uno o más partners - y la gestión de las interdependencias sobre bases contractuales presentará costes en términos de controles y garantías ${ }^{14}$. En el caso en que estas últimas sean también de carácter reiterado, de duración indefinida y caracterizadas por la incertidumbre estructural, existe un terreno fértil para el desarrollo -a través de la asunción de la capacidad de control y la internacionalización organizativa de las transacciones- de formas de coordinación jerárquica: la visión del mercado como multitud de actores independientes ligados por relaciones de tipo contractual cede a la visión de una población de organizaciones complejas (definibles como estructuras de gobierno y no como funciones de producción), insertadas en redes de relaciones. Redes que son en algunos casos horizontales y de intercambio, en otros son definidas por relaciones de adquisición y control, y en otras actúan mediante formas híbridas de intercambio y control. Centralización y asimetría se revelan como características de complejos organizativos tanto en el sector público, como en el sector privado (E. O. WiLliamson, 1975: 8), incluso si el carácter analítico de la teoría permite re-comprender como casos particulares la especificidad de las organizaciones públicas, por ejemplo en el tipo de compromisos que son activados en el curso de su desing (E. O. Williamson, 1994).

Todas las formas jerárquicas requieren formas de planificación: una vez determinada la internacionalización de los nexos de las transacciones con el fin de reducir la incertidumbre, el conjunto de estos nexos no puede ser dejado a formas de "acomodamiento" descentralizado, sino que requiere una descripción centralizada del contexto, de sus posibles evoluciones, incluso de los instrumentos legitimados para hacer frente a tales evoluciones y para resolver las tensiones internas creadas. Toda organización, de cualquier tipo, debe tener, y en efecto usualmente tiene, una red consolidada de prácticas, formales o informales, que equilibran los costes de transacción internos y externos, se esfuerzan por prever las variaciones y ligarlas a las relaciones establecidas entre objetivos, condiciones y recursos (internos y externos) con el fin de garantizar al menos un mínimo de congruencia. Por tanto, la planificación asume el carácter de un coste de transacción ex ante, eficiente en la medida en que contribuye a reducir la incertidumbre y los riesgos asociados con el conjunto de las transacciones (E. O. Williamson, 1981).

Es sabido que la teoría de los costes de transacción presenta también una serie de dificultades analíticas y metodológicas (M. Granovetter, 1985). A pesar de estos límites, sin embargo, representa un importante terreno de integración entre elementos sociológicos y económicos, y permite a la teoría de la pla- 
nificación ir más allá de la tradicional justificación en términos de bienes públicos. (J. M. BRYson, P. S. Ring, 1990). Esta perspectiva elabora para la teoría de la planificación un enganche -en términos de análisis estructural- entre el análisis del proceso de planificación y el análisis organizativo, reformulando la confrontación entre coordinación de mercado y coordinación jerárquica en términos de trade-offs entre diversos problemas de gestión de las relaciones sociales. Por otra parte, contemplar el proceso de planificación en términos de costes de transacción permite indicar autónomamente los límites de la misma planificación: el tránsito de la planificación a la adquisición descentralizada (y viceversa), dependiendo de la relación entre costes de transacción y costes de coordinación jerárquica, podrá tener ritmos tanto expansivos como de contracción: estructuras de interacción jerárquica podrán -por ejemplo, a continuación de los cambios tecnológicos- convertirse en pasibles de coordinación descentralizada, y áreas de intercambio de mercado podrán ir al encuentro de procesos de integración y concentración.

\section{Los contextos de la planificación: jerarquías, relaciones inter- organizativas y redes}

Si se adopta la teoría de los costes de transacción para definir las conexiones entre el proceso de planificación y las formas de coordinación jerárquica, es preciso tener presente las diversas formas de planificación en relación a los diferentes problemas de coordinación existentes en un sistema social. Dicho en otros términos, el análisis del proceso de planificación requiere una estrecha integración con el análisis de las modalidades de control interno y externo de las organizaciones planificadas. En esta clave parece útil distinguir entre:

1. Los procesos de planificación que tienen lugar en el interior de una organización, generalmente definidos en la literatura como procesos de planificación estratégica.

2. Los procesos de planificación que se producen en el nivel de un sistema interorganizativo, tradicionalmente denominados coordinative planning 15 .

3. Y los procesos de planificación activados por organizaciones -cuyo principal recurso es la capacidad legitimada de modificar el ambiente institucional de las transacciones y de definir $e x$ ante la alternativas de acción practicables- encaminados a definir el "territorio" de las transacciones para poblaciones de organizaciones y de familias, generalmente definidos en la literatura como procesos de comprehensive planning o master planning ${ }^{16}$.

Antes de revisar brevemente las características específicas de estos contextos de la planificación, es importante precisar tres puntos cruciales relativos a los límites de las organizaciones, al significado de los sistemas interorganizativos y al rol del ambiente institucional.

Por lo que se refiere al primer problema, es necesario ser conscientes de que la definición de los límites de una organización es normalmente muy arbitraria (E. R. ALEXANDER, 1992a). Más allá de ciertos umbrales de complejidad, organizaciones como las empresas con muchas divisiones o las agencias gubernamentales pueden ser tratadas por un observador como agentes unitarios o como poblaciones vinculadas de sub-organizaciones, posiblemente en competencia recíproca (J. S. LUKE, 1991). Si se acepta el énfasis institucional en las relaciones de gobierno, en algunos casos será relativamente fácil identificar el actor o los actores que poseen directamente el control, y evaluar la coherencia de la configuración organizativa y de las estrategias planificadas en relación a sus intereses; en otros casos, sin embargo, esta definición basada en el control directo se revelará inadecuada para comprender efectivamente la estrategia organizativa y la decisión requerirá la inclusión de categorías de actores con diversa denominación interdependientes con los insiders: los directivos, los proveedores, los empleados, los entes de control, los mismos clientes ${ }^{17}$.

Esta perspectiva es definida en los términos del tránsito del planteamiento para stockholders -la realidad de la organización debe evaluarse en referencia a los intereses de aquellos que la poseen- a la identificación de los stakeholders -la realidad debe evaluarse en relación a los grupos o categorías que tienen intereses interdependientes en la actuación de la organización ( $R$. E. Freeman, 1984; I. Mitroff, 1983). Esta concepción de los límites de las organizaciones permite revelar cómo la conexión entre jerarquía y planificación no sólo representa una chance de racionalización, sino también un instrumento de gestión de la identidad operativa. Es a través de la planificación como, implícita o explícitamente, una organización define quién está "dentro" y quién está "fuera", qué es "central" y qué es "periférico", hacia dónde se está "caminando" y de dónde "se viene". Identidad y control son dos caras de la misma moneda (H. C. WhITE, 1992).

Por lo que respecta al significado de los sistemas interorganizativos, es necesario subrayar que las diferentes formas de organización están comprendidas y todas presentan a su vez costes de transacción. Las islas de coordinación jerárquica están insertadas, por ejemplo, en contextos de interdependencia. En algunos 
casos, la gestión de las interdependencias entre diversas organizaciones tendrá un carácter preferentemente descentralizado de competición y de intercambio; en otros actuarán redes de coordinación informales, basadas en la costumbre, en las experiencias precedentes o quizás en la participación de los directivos como si fuesen un "clan", según los casos estudiados por W. OucH (1980); incluso en otros casos, donde los costes de transacción son muy altos, las organizaciones tenderán a desarrollar relaciones jerárquicas, que pueden ir desde el control directo (a través, por ejemplo, de adquisiciones o fusiones) a formas confederadas basadas en la institucionalización de organismos de coordinación comunes, con identidad propia y propias formas de planificación (R. E. AleXander, 1991; D. L. Rogers, D. A. Wettens y otros, 1982).

Por lo que respecta al ambiente institucional, es necesario recordar que ninguna organización y ningún sistema interorganizativo actúa en un vacío neumático. La configuración institucional (el orden más o menos fáctico que gobierna las relaciones interorganizativas) no sólo puede definirse en relación a las transacciones entre los actores, también en relación al ambiente institucional, el conjunto de normas que regulan las transacciones ${ }^{18}$. Las relaciones entre este ambiente institucional y las organizaciones son bi-direccionales: por una parte, el ambiente institucional constituye un parámetro independiente de la planificación -ya que un cambio en las reglas del juego comporta una re-organización del mismo juego-; por otra, el conjunto de los sistemas organizativos actúa estratégicamente respecto a tales reglas, tratando de provocar el cambio, de encauzarlo en determinadas direcciones o de minimizar el impacto. En el caso en que el ambiente institucional esté ligado a instituciones especializadas (por ejemplo, el sistema del Derecho), esta tensión relacional podrá traducirse en una forma de sistema interorganizativo con particulares características ${ }^{19}$.

Estas consideraciones muestran que la distinción entre planificación estratégica, coordinative planning y master planning, debe entenderse como una distinción relativa al contexto de las interacciones, no como una distinción entre técnicas, realizada en términos instrumentales. Estas formas de planificación son -al menos en cierta medida- interdependientes; los mismos sujetos pueden implicarse en una multiplicidad de relaciones diversificadas y los mismos "objetos" de la planificación, a menudo, pueden ser considerados por diversos actores en términos diferentes; la articulación de un proceso de planificación requiere un proceso de determinación, con grados diferentes de autonomía, de la propia unidad de referencia - del propio cliente ${ }^{20}$ - no sólo respecto a los "mercados" en que está insertada, sino también respecto a la red de todos los procesos de planificación (y de las expectativas sobre los cambios introducidos en cada uno de éstos). Esta distinción también puede asumir el carácter de transformación dinámica: por ejemplo, dada la dinamicidad de las relaciones de gobierno, la planificación estratégica (centrada en la singular relación organización/ambiente) puede llevar a la implementación de formas de coordinación jerárquica con otras organizaciones; formas de coordinación interorganizativa pueden realizarse en organizaciones independientes; cambios en el ambiente institucional pueden orientarse a la restricción en el ámbito de las posibilidades de coordinación jerárquica (por ejemplo, privatizaciones; normativas anti-trust) o a la expansión.

Sólo después de haber aclarado la interdependencia entre estas formas se puede proceder a la simplificación de un trato independiente para cada una de ellas.

\section{Planificar en una organización}

La planificación estratégica, nacida en el campo militar (y en los procesos de construcción de los Estados nacionales), se ha ido introduciendo de forma creciente en el mundo de las organizaciones económicas de medianas y grandes dimensiones, hasta llegar a convertirse en una forma de planificación necesaria en todas las organizaciones complejas, públicas o privadas (J. BRAKER, 1980). Su contexto de referencia está constituido por una dirección organizativa encaminada a afrontar la necesidad de peticiones contrastadas (que requieran la introducción de un orden en las prioridades), de previsiones sobre la alteración futura del equilibrio de los recursos y de los vínculos a los que la organización esta adaptada, de desafíos a la legitimidad de la misma organización, de crecimiento o insatisfacción hacia el nivel de coordinación interno o de cambios en curso en el ambiente y en la configuración institucional (J. M. BRYSON, R. C. EINSWEILER, 1988).

La planificación estratégica puede ser definida como el intento de articular dos diferentes descripciones de la organización (actual y futura) y ligarlas mediante la respuesta a cuatro preguntas:

1. ¿Dónde queremos ir? (La "misión” de la organización).

2. ¿Cómo haremos para llegar? (Las estrategias).

3. ¿¿De qué recursos tenemos necesidad? (El "budget”).

4. ¿Cómo nos orientamos? (El sistema de controles) ${ }^{21}$.

Este intento requiere un análisis (o un examen sistemático) de la situación organizativa en referencia a la misión (los objetivos) de la organización y al estado del ambiente - "los clientes" y otros stakeholders, concurrentes o aliados, cambios institucionales y tecnológicos (aquellos que son definidos como las "amenazas" y las "oportunidades" que se presentan)-. Este análisis es 
tanto una evaluación (del estado actual), como una previsión (sobre la evolución futura). De este análisis emerge la posibilidad de evaluar la organización en términos de puntos de fuerza y de debilidad, en la confrontación tanto con la situación actual como futura (prevista y/o deseada), y de definir respecto a estos dos puntos los sentimientos de cambio y las prioridades operativas.

Este esquema general es modulado en términos muy diversos, según el tipo de problemas afrontados y el tipo de contexto en el que el proceso de planificación debe intervenir. Existen planteamientos compactos y sistemáticos centrados en la asunción de un control fuertemente unitario (por ejemplo, Harvard Policy Model) y modelos que se concentran en una pluralidad de demandas internas y externas en las confrontaciones de la organización (Stakeholders Models); modelos de procedimiento encaminados a definir estrategias de coordinación trasversales respecto a los niveles jerárquicos y a las funciones (por ejemplo, sistemas de planificación estratégica) y modelos centrados en la identificación de criterios objetivos para la identificación de prioridades (por ejemplo, los llamados modelos de maletín); modelos basados en la introducción sistemática de innovaciones y modelos que privilegian continuos desarrollos de focalización de procesos incrementativos (J. B. QUINN, 1980)22.

La planificación estratégica permite tratar conjuntamente previsión y organización, con modalidades que consienten la posibilidad futura de discontinuidades, cambios, y diferentes tipos de imprevistos (I. AnsofF, 1981). Mientras que algunos planteamientos de previsión son rígidos, la planificación estratégica permite un ejercicio de previsión más compatible con adaptaciones flexibles al cambio del ambiente (incluidos los cambios introducidos por la propia acción organizativa). En segundo lugar, la planificación estratégica tolera una discusión no solo de lo que una organización hace -y cómo debería hacerlo-, también por qué y si tal organización debe hacerlo. En fin, la planificación estratégica permite un aprendizaje comparativo de los procesos comunicativos entre organización y ambiente.

Sin embargo, estos elementos no deben llevarnos a pensar que la planificación estratégica es un proceso necesario e insustituible. Por lo que respecta al carácter automático, se puede señalar como un proceso de planificación requiere la presencia de una leadership interesada en implementar la perspectiva que emerja y que esté en condiciones de hacerlo. Esta condición no hay que darla por descontada en el mundo real: muchas organizaciones pueden estar tan fragmentadas que tan sólo permitan estrategias incrementativas; otras actúan en tales condiciones que están más próximas a ser una especie de "federación" de sub-unidades que no a organizaciones unitarias; otras pueden encontrarse en la situación de tener que activar una decisión estratégica para la adaptación táctica. En otras palabras, el nivel al que debería ser conducido el proceso de planificación puede ser bien diverso del formalmente establecido para gestionarlo. En otros casos, en fin, las exigencias que motivan el inicio de un proceso de planificación estratégica estás ligadas no a la voluntad de implementar las conclusiones, sino al efecto de imagen que puede producir en el exterior o en el interior. El proceso de planificación estratégica, entendido como un proceso especializado y formalizado, no puede ser considerado "no sustituible". Es sabido que muchas organizaciones actúan no mediante procesos de planificación formalizados, sino a través de decisiones carismáticas de uno o más leaders que no aceptan el debate y discusión de la propia forma organizativa y afrontan separadamente las oportunidades y las amenazas que se presentan.

En síntesis, la planificación estratégica -como proceso formal- se presenta como una alternativa realizable en presencia de desafíos y cambios ambientales (comprendiendo también en tal conjunto la opinión de los stakeholders) suficientemente profundos como para motivar los costes y los riesgos del proceso, pero limitados para no llegar a asumir como resultado probable la desaparición de la organización o un desafío consistente a su dirección. En muchos otros casos -donde la organización esté efectivamente fragmentada y existan dificultades para identificar una leadership unitaria-, el proceso de planificación tenderá a asumir el carácter de una forma mixta entre planificación estratégica y coordinative planning.

\section{La planificación de los sistemas inter-organizativos}

La perspectiva del proceso de planificación se presenta en términos bien diversos cuando el contexto de referencia no es una singular organización, sino un conjunto de organizaciones no unificadas bajo un mismo control. Un buen ejemplo es el caso de las asociaciones de organizaciones (una asociación empresarial o una asociación de sector) o parcialmente investidas por cualquier forma de poderes derivados de los propios participantes ${ }^{23}$. Aunque es difícil evaluar cuantitativamente la relevancia de tales formas en las sociedades modernas, seguramente es discreta y, según muchos autores, en continuo crecimiento (D. A. WETTEN, B. BOZEMAN, 1991).

En efecto, el desarrollo de los sistemas interorganizativos es una consecuencia del incremento de la interdependencia societaria, interdependencia que produce un crecimiento de los problemas de coordinación tanto entre poseedores de recursos similares pero insuficientes respecto al objetivo propuesto, como entre poseedores de recursos diferenciados integrables en nuevas direcciones. En estos casos, incluso donde exista, la presencia de un coordinador -o de una unidad de coordinación- no es segura- 
mente equivalente -en el sentido funcional- a la de un superior jerárquico en una organización unificada: por ejemplo, los poderes de definir misiones, objetivos y prioridades están sensiblemente limitados por los intereses estratégicos de las organizaciones participantes, y la relación de principal-agente a menudo está caracterizada por una pluralidad de "principales" con intereses y perspectivas diferenciadas y a su vez en concurrencia. Generalmente, por otra parte, tales realidades gozan de un reducido poder de sanción, a menudo mediante la reducida capacidad de promover y coordinar las sanciones de una parte relevante de los actores participantes hacia los actores recalcitrantes.

En estas condiciones, el problema principal de la planificación es identificar estrategias que permitan una distribución relativamente satisfactoria de los objetivos entre las unidades participantes -de forma compatible con sus misiones y con sus estrategias (desarrolladas autónomamente) - y asegurar el compromiso de estas organizaciones hacia tales objetivos, sin recurrir a cargas o sanciones que hagan preferible una siempre posible solución de tipo exit. Éste es el campo del coordinative planning. Esta forma de planificación está caracterizada por un énfasis en la actuación de estrategias coordinadas (respecto a la producción de estrategias propias) y en la individuación, gestión y desarrollo de convenciones y de otras modalidades de coordinación que sean compatibles con las estrategias de las singulares organizaciones participantes. En otras palabras, la fuerza potencial de esta forma de planificación reside no en la capacidad de amenazar con sanciones negativas, sino en la capacidad de ofrecer incentivos y potencialidades de gestión de las zonas híbridas entre jerarquías y redes $^{24}$. En consecuencia, la forma de planificación dominante es la negociada: algunos objetivos -generalmente asumidos de forma genérica- son desarrollados y contextualizados en referencia a los acontecimientos que suceden, utilizando las interacciones previstas, la identidad colectiva y los escenarios previstos contemporáneamente como instrumento de coordinación y como terreno de contratación. El planificador, aunque esté legitimado funcionalmente en términos de competencias sustanciales, actúa en el proceso principalmente como mediador y como "caja de resonancia” para la definición del problema y de las agencias (L. E. Susskind, C. OzaWA, 1984). En el plano de las propuestas, los modelos de planificación más pertinentes son los múltiples, basados en la definición de una pluralidad de objetivos alcanzables con diferentes medios, es decir, que permitan maximizar la posibilidad de fitting entre objetivos del sistema interorganizativo y objetivos de las singulares organizaciones 25 .

Otra modalidad de planificación a adoptar en el nivel de los sistemas interorganizativos está constituida por la llamada planificación indicativa (J. A. MEADE, 1970). Esta forma de planificación promueve la reducción de la incertidumbre mediante la puesta en común de las informaciones que permitan a toda organización participante reducir los márgenes de imprevisión de los otros actores (incluidas, en cuanto participantes, las instituciones reguladoras). Esto se refleja, para las unidades participantes, en la elaboración de las propias decisiones, reduciendo los costes de intelligence y de complejidad computacional. La complejidad de esta forma de planificación no debería sin embargo minusvalorarse. En primer lugar, para ser utilizables, las informaciones disponibles deben ser criticadas -respecto a la elaboración de las estrategias- y monopolizadas (imposibles de conseguir mediante otros canales). Como consecuencia, estos procesos de planificación requieren particulares procesos de observación -desde el punto de vista de las organizaciones participantes-, de concertación -sobre el tipo, la calidad y el detalle que los participantes están dispuestos a hacer públicos- y de sanción -contra la tentación de los participantes a comportarse como free riders-. Cuando los factores clave de la salida del proceso son referentes a la disponibilidad de informaciones sobre las actividades de las instituciones reguladoras o al control de las sanciones vinculantes, la planificación se convierte en una asignación de los poderes públicos y se transforma en una forma de master planning.

Los sistemas interorganizativos pueden parecer inestables y no faltan autores que los consideran formas de fricción, destinados a evolucionar hacia la fusión en una organización unitaria 0 , alternativamente, hacia la disolución. Sin embargo, según otros, los sistemas interorganizativos -y el coordinative planning- representan elementos fundamentales de la gestión de las interdependencias, capaces de transformar -si están adecuadamente gestionados- sus debilidades en fuerzas (D. A. WhetTen, B. BOZEMAN, 1991).

\section{Master planning y planeamiento institucional}

El Master Planning es la forma de planificación más conocida y discutida en la teoría de la planificación. Aún más, con frecuencia ha sido considerada la forma de planificación tout court. Una organización -generalmente un ente público al que se atribuyen poderes sobre contextos difusos, por ejemplo de tipo territorial- realiza una serie de previsiones (respecto a un área geográfica o a un sector social) e introduce decisiones que asumen un carácter vinculante, modificando el ambiente institucional en el que actúan los actores ${ }^{26}$. Este conjunto de elecciones opera en diferentes direcciones:

- Estimular el desarrollo del ambiente en direcciones intencionalmente seleccionadas, pero también contribuir a la capacidad de adaptación respecto a los cambios independientes (actuales o previstos). 
- Distribuir recursos (o gestionar la reducción de los mismos) en utilizaciones independientes, pero también desarrollar nuevos proyectos.

- Incentivar comportamientos innovadores, regular las interdependencias entre conjuntos de actividades autónomas y reducir comportamientos perjudiciales (J. FriEDMAN, 1967).

Esta amplia gama de elecciones institucionales es perfectamente compatible, en muchos casos indispensable, para la existencia de una sociedad libre, y están legitimadas por el hecho de constituir, en contextos con fuerte interdependencia, una precondición misma de la racionalidad de los actores participantes ${ }^{27}$.

El contexto de esta forma de planificación, es sin embargo sustancialmente diferente de los que han sido analizados hasta ahora. El ambiente de este proceso está compuesto tanto por la estructura interna de la organización, por otras organizaciones -sobre las que no se poseen derechos de control- como por las familias $^{28}$. Estos referentes están a su vez ligados e interdependientes entre sí mediante una pluralidad de formas de relación (intercambio, jerarquías, redes, comunidad), pero también diferenciados en términos de intereses, valores y prioridades. En términos internos, la organización planificadora controla un recurso crucial: la capacidad de emitir decisiones vinculantes, modificando el ambiente institucional de los actores.

Dadas estas características, la teoría clásica de la planificación atribuía al proceso de master planning un carácter casi geométrico: al planificador se atribuía el objetivo de identificar "objetivamente" los problemas sociales y de realizar científicamente las previsiones de las que derivar -de forma también objetiva- algunas recomendaciones a transmitir a un director político que, una vez aprobadas, las asignaba a una estructura burocrática contemplada exclusivamente "operativa" y hetero-dirigida. Es esta concepción, más que la planificación en cuanto tal, la que se presenta inadecuada y fuertemente problemática tanto en términos de eficiencia como de legitimación sustancial. Desde el perfil del planteamiento, esta concepción se basaba en la utilización inapropiada de formas de planificación estratégica que daban por descontada la unidad decisional de la agencia pública, minusvalorando la complejidad y fuerza de las configuraciones presentes en el ambiente de la organización, sobrevalorando las posibilidades de aplicación de la coordinación mediante decisiones jurídicamente vinculantes y olvidando sustancialmente el rol de la implementación (G. Benvenist, 1991; J. Friedman, 1987; J. M. Bryson, R. C. EINsweiler, 1988).

Cada uno de estos límites es imputable a una visión inadecuada de la relación entre el proceso de planificación y su contexto social. A nosotros nos interesa centrarnos en la interconexión de tres figuras clave: los planificadores, los que deciden y las burocracias (más en general, aparatos de implementación).
Por lo que respecta al planificador, es necesario ser conscientes de que en los contextos de master planning, la relevancia del conocimiento y de la competencia técnica jamás pueden separarse de las operaciones principales de la acción política: argumentar y contratacar. En los procesos de planificación, el conocimiento técnico no solamente es un recurso operativo: adquiere un significado como elemento de vínculo o de recurso para las capacidades de los singulares actores para argumentar y contratacar. Tener presente esta función del conocimiento es fundamental, por ejemplo, para comprender los motivos por los que el marco o la narrativa en los que se elabora la planificación termina por constituir la principal contribución del planificador al proceso de decisiones (F. FISHER, J. Forester, 1993; E. RoE, 1994). Por otra parte, lejos de ser unidireccional, la relación conocimiento-política es sustancialmente bidireccional: la transferencia del conflicto al mismo terreno del conocimiento "científico" es una opción siempre posible (mediante modalidades que van desde la elaboración de previsiones alternativas a la pura y simple deslegitimación de los planificadores). En fin, las modalidades productivas y organizativas del mismo conocimiento a menudo permiten hacerlo "negociado", antes incluso de que exista y se transmita al que tiene que decidir (M. S. FELDMAN, 1989). Dados estos elementos, la idea del planificador como "mecánico" o "terapeuta" se revela sustancialmente ilusoria, y es preferible su comprensión como un consultor profesional reflexivo (A. SCHON, 1983): planificar racionalmente quiere decir planificar políticamente (J. FORESTER, 1989).

Por lo que respecta a la decisión política, al que decide la realidad se presenta muy diferente de la que podría hipotizar a partir de su poder de decisión definido formalmente. En primer lugar, tal decisión podrá a su vez ser desafiada jurídicamente, y en casos de relevante complejidad es difícil minusvalorar las amplias posibilidades de error y especialmente de retrasos; la ubicación estructural del que decide permite (generalmente) márgenes de autonomía para toda singular coalición de intereses, pero no para todas al mismo tiempo; los tiempos de "retorno" de la propia inversión política pueden ser muy diferentes de los necesarios para desarrollar la intervención propuesta. Por otra parte, si es verdad que el que decide controla el recurso constituido por las posibilidad de emitir decisiones vinculantes legitimadas, también es cierto que frecuentemente actúa desde el interior de una definición institucional genérica (¿cuál es -por ejemplo- la "misión" de un ayuntamiento?) y que la selección de los stakeholders difícilmente puede derivarse de ésta en términos de inferencias (en una ciudad, ¿quién es central y quién es periférico?, en presencia de conflictos distribuidos, ¿qué interés es el prioritario?). En efecto, a menudo el ente planificador corresponde a la clásica descripción que G. Aluison (1971: 67) dio del gobierno americano en su estudio sobre la crisis cubana: "un gobierno consiste en un conglomerado de organizaciones semi-feudales, aliadas de 
forma laxa, cada una con su especifica vida. Los leaders están formalmente por encima de tal conglomerado (...). El gobierno debe ser interpretado no tanto en términos de elecciones deliberativas, como de producto de las grandes organizaciones funcionales según determinadas estructuras de comportamiento". En otras palabras, el contexto de las decisiones requiere no solamente planificación estratégica sino, también, y sobre todo, coordinative planning y planificación indicativa.

Por lo que respecta al aparato de la implementación, también es sabido que decisiones de largo alcance pueden ser totalmente ineficaces si son incompatibles con las lógicas de funcionamiento de los aparatos de implementación a los que es delegada la actuación. En primer lugar, los aparatos de implementación son muy diferentes de los modelos mecánicos que han dominado durante bastante tiempo en la teoría de la burocracia: el equilibrio de los poderes internos, la competencia entre las funciones, la evaluación de las prioridades generalmente son diferentes entre órganos de decisión y estructuras de implementación (B. HJERN, D. PorTer, 1981). Concretamente, no hay que olvidar que el estado del aparato de implementación es una variable relevante de los juegos de interdependencia que definen el contexto: por ejemplo, la eficiencia organizativa de la organización -su capacidad efectiva de sancionar eficazmente a los trasgresores de la decisión tomada- constituye en buena medida parte de la "credibilidad" de la organización y de la motivación de los otros actores presentes y comprometidos en la participación en el proceso de decisiones más que en evadirlo continuamente. Entes planificadores de este tipo -incluso a causa de la pluralidad de finalidades ya indicada- a menudo están comprometidos en una pluralidad de formas de coordinación gestionadas de forma diferente. Un ente planificador, por ejemplo, puede actuar a menudo mediante políticas de dirección en un sector, a través de una planificación indicativa en otro y promoviendo intervenciones de red en un tercero. Y el conjunto de los problemas que tal organización gestiona puede en algunos casos ser tratado según una planificación racional, en otros mediante formas incrementativas, y en otros incluso a través de formas de intervención residual. La evolución de todo nuevo proceso de planificación no podrá ignorar este conjunto plural de experiencias, estilos y competencias ya disponibles (y que pueden eventualmente constituir "el problema" que motiva el nuevo proceso).

Ignorar tales características del contexto, para mantener una rígida división entre "experto" y "gestor" no constituye un incremento de las posibilidades de racionalidad del proceso de decisiones, sino que, al contrario, facilita un uso puramente ritual. Insertar explícitamente la dimensión comunicativa y política en el interior del proceso de planificación parece que es preferible tanto para mantener el carácter de actividad práctica del proceso de planificación, como para obtener una descripción empíricamente adecuada de lo que los planificadores hacen efectivamente (J. FORESTER, 1989).
Por tanto, desde su origen, el master planning está constituido -desde el punto de vista del ente planificador- tanto por elementos de la planificación estratégica, como del coordinative planning. Desde algunos aspectos, el ente planificador está investido de poderes tales para actuar estratégicamente a partir de una propia determinación de los objetivos (tratando al resto de organizaciones presentes en el ambiente como objeto previsto pero no como partner); desde otros, por ejemplo en la promoción de determinados desarrollos o en la mediación entre intereses funcionales confluyentes, la organización se encontrará con tener que actuar a través de la promoción de formas de coordinative planning, utilizando principalmente la propia capacidad de terciar como incentivo y los propios poderes de decisión como "amenazas" en última instancia. En este contexto el proceso de planificación asumirá el carácter de planteamiento institucional, entendiendo con este término el planteamiento de formas de coordinación concebidas "más que como estructuras organizativas (...), como procesos que incluyen incentivos para la generación del necesario compromiso, la legitimación del contexto especifico, la monitarización y la sanción de las acciones deseadas, y las modalidades apropiadas de resolución de los conflictos" (E. R. ALEXANDER, 1994: 345).

La calidad y la fuerza de una intervención de planificación en este contexto dependerá no sólo de la calidad del análisis de previsiones y de la implementación, también de la capacidad de integrar y racionalizar este conjunto de prácticas con la configuración evolutiva del ambiente institucional y con las prácticas de planificación ya realizadas en los diversos sectores y niveles.

\section{El proceso de planificación como estructura de interacciones}

Sin embargo, la sociabilidad del proceso de planificación no se agota en su relación "externa" con el contexto social en el que interviene. Igualmente importante es el carácter social "interno": el proceso de planificación es una interacción estructurada entre participantes insertados en roles diferentes -o por lo menos analíticamente diferenciables- que controlan diversos tipos de recursos y son objeto de expectativas diversificadas. Por ejemplo, desde el principio de estas páginas se ha diferenciado entre dos roles fundamentales del proceso de planificación: el "planificador" -definido en términos de posesión profesional de metodológicas y técnicas de previsión y organizativas- y el "que decide" -definido por la capacidad legitimada de actuar- con diferentes 
grados de control sobre las elecciones vinculantes. La introducción del rol de implementador - del "burócrata" o del "funcionario" que controla la operatividad interna de la máquina organizativa- rápidamente ha sido necesaria ${ }^{29}$. Estos tres roles definen la estructura de interacciones mínima de un proceso de planificación y el orden de interacciones que se establece entre estos tiene un cierto grado de autonomía del contexto social de referencia.

En diversos puntos, a lo largo de las anteriores páginas, se ha revelado cómo en la teoría clásica de la planificación -y a menudo en el sentido común de las organizaciones y de los profesionales participantes- esta interacción es interpretada como una división del trabajo mecánicamente predeterminada: el que decide presenta al planificador un problema relativamente bien definido y recibe una respuesta, formulada en el lenguaje del conocimiento "objetivo" que reduce el ámbito de la elección a pocas alternativas bien razonadas. Una vez seleccionada la alternativa preferida, ésta pasa al "burócrata" que procede a implementarla de la mejor forma posible, activando las necesarias estructuras organizativas y los oportunos incentivos para el personal. Este último actúa de forma bien definida y en coherencia con los objetivos prefijados.

Esta descripción no sólo es inexacta en referencia a los contextos concretos, también es inadecuada como guía normativa en el comportamiento de los mismos planificadores (A. SCHON, 1983; S. J. Mandelbaum, 1986). Lo que emerge del análisis de los contextos y de las experiencias es un modelo de interacciones del proceso de planificación caracterizado por la continua negociación de una perspectiva común a partir de la constante búsqueda de compatibilidad entre perspectivas y criterios considerados diferentes (M. S. Feldman, 1989). Más que un proceso de derivación lógica, un proceso de planificación recuerda una interacción con doble contingencia, en la que finalidades diferenciadas actúan desde el interior de un cuadro que anticipa selectivamente los vínculos y los recursos controlados por el resto de actores al realizar las propias selecciones.

Si la visión "técnica" del proceso de planificación se presenta inadecuada para la reconstrucción del proceso de planificación, muchos de los análisis realizados en estas páginas permiten afirmar que también es incompleta para la identificación de los actores que contribuyen a configurarlo. Por ejemplo, revisando los límites de la organización ha emergido el tema de los stakeholders, tratados como categorías de intereses difusos cuya identificación es un componente crucial del proceso de planificación. Se puede distinguir aquí entre:

a) Stakeholders organizados (por ejemplo, las asociaciones de categoría, los sindicatos) o representantes de aparatos institucionales consolidados.

b) Categorías difusas y no organizadas (consumidores, ciudadanos, etc.) cuya única representación está constituida por "representantes" no formalmente delegados (por ejemplo, grupos ambientales, comités cívicos, asociaciones de consumidores).

Estas formas de representación de los stakeholders poseen características comunes: no son "adversarios" políticos o "competidores" económicos del que decide, incluso pueden aliarse con éste, y están estructuralmente interesados no sólo en la capacidad de influenciar el proceso de planificación, también pueden ser reconocidos preliminarmente como actores estables (ya que extraen de tal participación un motivo de legitimación en las confrontaciones con los propios miembros). Si se pasa revista a las reglas institucionales del proceso de planificación -especialmente en el caso de organizaciones públicas- es fácil revelar la existencia de numerosos canales que van transformando en muchos sectores a los grupos de intereses, especialmente del primer tipo, en actores estables del proceso ${ }^{30}$. Algunos teóricos de la planificación identifican en esta inclusión -concretamente en el caso de los "nuevos" movimientos- un fenómeno positivo, susceptible de conducir a una mayor coordinación, a una menor dependencia de los intereses consolidados y a una menor incertidumbre informativa (J. FrIEDMAN, 1967; 1987: 343-412). Sin embargo, otros sostienen que la presencia de grupos de presión -más fácilmente integrados por reivindicaciones negativas que por propuestas positivas- en el proceso de planificación comporta un incentivo para los que deciden con el fin de mantener internamente el statu quo consolidado (P. HALL, 1980).

Independientemente de la evaluación del fenómeno, es difícil negar que el "representante de intereses", verdaderos o supuestos, actualmente es un actor relevante de los muchos procesos de planificación, y que la previsión y gestión del proceso de planificación frecuentemente debe tener en cuenta sus reacciones (M. DeAR, 1992). La inserción de los "representantes" también conlleva la atención sobre un hecho: la estructura de la interacción incluso es definida por algunos actores "invisibles", los convidados de piedra, que -no presentes en la interacción- sin embargo, constituyen el referente para la anticipación de la recepción de la misma intervención. Podemos considerar entre estos convidados de piedra a dos potenciales categorías:

a) Los stakeholders reconocidos como tales, pero que no se reconocen en los "portavoces" de la categoría incluidos en la consulta (y que pueden actuar negativamente no sólo en el proceso de planificación, también en la inclusión del mismo "portavoz").

b) Y las categorías que no son objeto de la prevención: un proceso de planificación puede entrar en crisis no sólo por errores internos, también por la interpretación de su existencia como distributivamente "injusta" 31.

En referencia a los convidados de piedra, el proceso de planificación es un acontecimiento de por sí comunicativo, indepen- 
dientemente de sus conclusiones específicas, que se refleja en la proyección de imágenes cargadas normativamente.

La definición del proceso de planificación como una interacción estructurada entre cuatro categorías de actores -los planificadores, los que deciden, los burócratas y los "representantes" de intereses- en presencia de un cierto número de actores invisibles que pueden activarse de forma imprevisible en el curso del proceso, parece corresponderse tanto con la evidencia empírica 32 , como con el pensamiento reflexivo de la planificación. Pensamiento que, merece la pena apuntarlo, en la última década ha empezado a considerar a algunos instrumentos de interacción -teoría de los juegos, técnicas de contratación, procedimientos de gestión de los argumentos- como parte integrante de la formación de todo buen planificador, contemplado no sólo como "analista", sino también como gestor de un proceso de interacciones (J. Forester, 1989; E. R. AleXANDER, 1994).

\section{Conclusiones}

En este trabajo hemos tratado de articular una perspectiva sobre el proceso de planificación que pudiese ir más allá de los límites del debate mediante la elaboración de una teoría más abstracta y menos vinculada a la rígida demarcación entre público y privado. Se ha argumentado que esta perspectiva sólo es posible mediante la reformulación del proceso de planificación como proceso intrínsecamente social, ligado a la gestión de las incertidumbres y de las identidades de los contextos de coordinación jerárquica.
A partir de esta re-elaboración ha sido posible articular las diferencias entre las formas de planificación (planificación estratégica, coordinative planning y master planning) no en términos de "métodos" o "técnicas", sino de diferencias relacionales, referentes a las diversas formas de coordinación jerárquica y a las diferentes constelaciones de poderes y de redes de comunicación que definen tales formas. Por otra parte, este planteamiento permite contemplar el proceso de planificación como una interacción estructurada entre actores diferenciados e interdependientes en presencia de un ambiente dinámico y de límites móviles.

Si se compara esta perspectiva con las promesas de la planificación "racional" propias de la teoría clásica, es difícil negar que la perspectiva articulada es al mismo tiempo menos nítida -en cuanto que contempla, por ejemplo, la previsión del proceso de planificación y su organigrama- y más débil -ya que al proceso de planificación se le hace depender de la integración de una pluralidad de intereses y perspectivas-. La misma identidad del planificador es sensiblemente transformada: de "consejero del príncipe" o grand commis d'état, pasa a ser un profesional cualificado que une a la competencia técnica capacidades comunicativas y negociadoras, y que vive insertado en un conjunto de relaciones caracterizadas por asimetrías múltiples.

El proceso de planificación, lejos de eliminar incertidumbres mediante el conocimiento "científico" se revela como un instrumento para gestionarlas. Sin embargo, en un mundo social caracterizado estructuralmente por la incertidumbre -que conviene no olvidarlo, es el correlato inevitable de la libertad de los actores-, no se ha dicho que un rol más incierto, y la modestia que tal incertidumbre facilita, no se presenten como más útiles y más indispensables.

\footnotetext{
* Departamento de Sociología. Universidad de Granada.

${ }^{1}$ Este punto está en el núcleo de otras definiciones más parciales de la planificación, como la realizada por J. FRIEDMAN (1987: 38): "la planificación es un intento de ligar el conocimiento cientifico y la técnica con la acción en el sector público".

2 Éste es el principal punto de intersección entre investigación social y proceso de planificación. Sobre este tema véase Di Nicola (1998: 225-243).

3 Sobre las formas, los estilos y el proceso de decisiones, véase G. BERTIN (1998:197-222).

${ }^{4}$ La importancia del nexo entre planificación e implementación, y la crítica a las perspectivas que lo han olvidado durante mucho tiempo, son tratadas en algunas partes de este artículo, concretamente en los apartados 3, 4 y 7. Sobre la importancia en el proceso de planificación de la fase de implementación, véanse D. PALUMBo (1987) y B. HJERM, D. O. PORTER (1981).

5 Sobre formas y funciones de la evaluación véase A. TRINIDAD (1996: 503-539).

${ }^{6}$ Afirmar que la planificación es un instrumento de racionalidad no quiere decir que la presencia de un proceso de planificación comporte de por sí que el curso de acción adoptado sea "racional" (desde el punto de vista de un observador externo). La planificación, tal y como es practicada empíricamente, puede ser errónea o constituir un elemento de irracionalidad organizativa. En términos normativos, sin
}

embargo, la planificación está estrechamente asociada a la acción racional.

7 Por sistema social se entiende "un sistema generado por todo proceso de interacción, sobre el nivel socio-cultural, entre dos o más actores. La interacción social es el proceso a través del cual el comportamiento o el cambio de estado entre los miembros del sistema influencia a) al estado del sistema y b) a los estados entre los actores y sus relaciones reciprocas" (T. PARSONS, N. J. SMELSER, 1956: 8). En este sentido toda forma de interacción estructurada es definible como un sistema social.

8 En realidad, la teoría sociológica ha superado desde hace algún tiempo el binomio Mercado-Estado, reconociendo la existencia y la importancia de otras modalidades de coordinación tales como las redes y las comunidades. Teniendo presente la finalidad descriptiva del presente párrafo, aludiremos a la distinción entre planificación y racionalidad de mercado, ya que es sobre tal distinción donde se concentra buena parte de la literatura.

9 Se debe subrayar que la existencia de bienes públicos implica la necesidad de una regulación pública, y no necesariamente de una intervención pública directa en su gestión (aunque -especialmente en el primer tipo- ésta sea la forma más difundida).

${ }^{10}$ En realidad, requiere un complejo y delicado conjunto de conciertos institucionales y de procesos de socialización. Véanse T. PARSONS (1937) y T. Parsons, N. J. SMELSER (1956). 
11 Una consecuencia particularmente interesante de la hegemonía de esta perspectiva se contempla en el nivel de las identidades profesionales. Los expertos, consultores y planificadores del sector público tienden a tener una vida profesional (en términos de asociaciones, publicidad, cursos de formación y actualización, etc.) diferenciada -y hasta hace cualquier tiempo casi incomunicada- con los expertos de planificación y consultores de las organizaciones privadas. Véanse B. CECKORAY (1986) y J. M. Bryson, D. W. Roering, (1988).

12 Si la teoría ha dedicado una amplia atención a los fracasos del mercado, el tema de los fracasos de las burocracias -y de la conexión entre planificación y organización- ha recibido una atención episódica y globalmente fragmentaria. El intento de transformar los documentos de planificación en libros de sueños ha encontrado pocos vínculos.

13 Por ejemplo, el coste de la información -en términos de coste y disponibilidad-, el coste de control -sobre la calidad de los bienes y de los servicios intercambiados- y el coste de las inversiones en elementos específicos de la singular transacción.

14 En la clasificación elaborada por el mismo WilLiamson (1981), éstos comprenden: costes de dirección, costes de gestión de la adaptación, costes de corrección de los errores, costes de construcción y gestión de las estructuras de gobierno y costes necesarios para garantizar la efectiva vinculación del partner al contrato.

15 Coordinative planning podría ser traducido como "planificación coordinante". Como se verá, el término coordinative planning no alude a la planificación ex ante de la coordinación, sino a la planificación "a través de" o "interna a la coordinación".

${ }^{16}$ También en este caso, la traducción literal conllevaría problemas. En el master planning se incluyen actividades tales como la planificación territorial o industrial realizada por agencias públicas o entres locales.

${ }^{17}$ La pequeña empresa con el control no dividido puede ser considerada un ejemplo del primer caso (incluso si su frecuente inserción en redes y cadenas productivas crea cualquier problema); las empresas multi-proveedoras de una demanda pública monopolista (ejemplo, la industria de la defensa) representan el caso más frecuentemente citado de la segunda modalidad.

${ }^{18}$ Es significativo que la economía de los costes de transacción haya descubierto (E. O. Williamson, 1994: 80) la definición de las instituciones realizada por T. PARSONS y N. J. Smelser: las instituciones son "modalidades con las que los modelos de valores de la cultura común de un sistema social son integradas con las acciones concretas de sus unidades en interacción reciproca mediante la definición de expectativas de rol y la organización de las motivaciones" (T. PARSONS, N. J. SMELSER, 1956: 102).

${ }^{19}$ Véase el apartado 7 de este artículo. Véanse también el debate recogido en R. J. Veld, L. SChaAp y otros (comp.) (1991) y los problemas afrontados en J. FORESTER (1989).

${ }^{20}$ Tomemos el caso de la planificación de una unidad insertada en el interior de una coordinación jerárquica. En este caso, la planificación deberá tener presente, más allá de las estrategias de los actores concurrentes, las estrategias de los que ordenan y de los subordinados (por lo menos como vínculo). En presencia de ambigüedades y de ciertos márgenes de autonomía, la literatura revela alguna duda sobre que tales estrategias siempre tendrán un carácter funcionalmente transparente (por ejemplo según la lógica de la descentralización "en cascada", en la que toda organización subordinada selecciona entre alternativas ya filtradas de las organizaciones que establecen las directrices -o del principio de subsidiariedad- en el que todo nivel superior se abstiene de intervenir en lo que puede ser planificado por un nivel inferior). La frecuencia de fenómenos tales como las prioridades secretas (Hidden Agendas) de agencias formalmente subordinadas, de la alteración de las misiones institucionales en curso de realización o-al contrario- de intentos de reducción de las autonomías formalmente adquiridas mediante instrumentos indirectos (por ejemplo a través de variaciones en las transferencias financieras o alteración de los criterios de evaluación de las performances) lleva a pensar que las interacciones entre los diferentes procesos de planificación pueden evaluarse posteriormente - dada la conflictividad inserta en la determinación de los límites- en términos de compatibilidad más que de coherencia. 21 Véase P. LORANGE (1980).

22 Un buen recorrido por los diferentes modelos de planificación estratégica y una evaluación de su utilidad por parte de los entes públicos puede encontrarse en J. M. BRYSON, W. D. RoERING (1988).

23 Otros ejemplos de esta realidad pueden ser considerados formas más delimitadas temporalmente y funcionalmente como, por ejemplo, un consorcio de organizaciones para la gestión de un proyecto común (J. M. BRYSON, R. C. EINSWEILER, 1991).

${ }^{24}$ La relación entre mercados, jerarquías y redes es uno de los campos de la sociología económica (y no sólo de ella) de mayor interés y relevancia para la comprensión de las sociedades modernas. Al ser imposible ofrecer en este contexto una descripción adecuada, aconsejamos que se vea W. W. PoweLL, L. SMITH-DoERr (1994).

25 Un ejemplo puede ser el "modelo del maletín" re-elaborado por el Center for Philadelphia Studies (1982) para dirigir las inversiones hacia determinadas zonas del área metropolitana.

26 Éste es el caso, por ejemplo, de un gobierno local cuando elabora un plan de infraestructuras o lleva a cabo una distribución planificada de la inversiones, o de un gobierno liberal que debe elaborar una política económica o industrial.

${ }_{27}$ En ausencia de un plan regulador, por ejemplo, no es posible actuar racionalmente en el mercado inmobiliario: el valor de una zona varía según las elecciones realizadas por los propietarios de zonas limítrofes, aumentando en algunos casos y disminuyendo drásticamente en otros. Por ejemplo, es difícil adquirir una casa si se ignora que sucederá en el futuro: jpodrá construirse una discoteca en las proximidades?, ¿todos los negocios de la zona perderán la licencia?, ¿los jardines públicos se transformarán en zona edificable al día siguiente de la adquisición?

${ }^{28}$ Desgraciadamente, en la literatura sobre la planificación, las diferencias entre organizaciones y familias son, salvo raras excepciones (por ejemplo, M. DEAR, 1992), sustancialmente olvidadas o bien los individuos y las familias son considerados más o menos equivalentes a organizaciones sui generis (por ejemplo, E. R. ALEXANDER, 1991, 1992a). Estas posiciones están ligadas a la asunción de que, en ausencia de un mínimo de organización, el potencial impacto de estos individuos en el proceso de decisiones y en la implementación será limitado cuando no nulo. Sin embargo, existen numerosos motivos, empíricos y conceptuales (véanse las conclusiones) para sostener que una teoría adecuada de la planificación no puede ignorar a estos "convidados de piedra".

${ }^{29} \mathrm{El}$ uso de comillas indica que los roles descritos no deben ser necesariamente asumidos como descriptivos de individuos concretos: el "planificador" puede ser un grupo de planificadores o un rol mental del que decide (o del burócrata). Y lo mismo vale para las otras figuras. Debemos tener presente que cada uno de los "roles" descritos también es parte de un retículo diferenciado de "iguales", cuya evaluación es activamente buscada: todo actor se preocupa de la opinión de los propios colegas y todo actor utiliza las propias pertenencias -que representan también la propia "reputación"como un recurso de interacciones. Cada una de estas redes no trasmite solamente informaciones técnicas y posibilidades comparativas, también modelos normativos de comportamiento: el potencial juicio de iguales a menudo es, junto con la experiencia, el criterio de guía principal de todo actor en sus relaciones con el resto de roles.

30 Especialmente en los contextos de master planning, donde son menores las barreras para el acceso y más fuerte la vulnerabilidad organizativa, la contratación, y donde la interacción con los "representantes" de stakeholders no organizados los convierte en una característica estable.

31 ¿Por qué el ente local debería ocuparse de la contaminación del agua (que no me afecta) y no de la contaminación acústica (que hace de mi vida un infierno); jpor qué interesarse por los parados y no por los que están a la búsqueda de un primer trabajo? En condiciones de escasos recursos, esta forma de conflictos distributivos forman parte de nuestra cotidianeidad.

32 Véanse los casos analizados por P. HaLL (1980). 


\section{Bibliografia}

Alemán Bracho, C., Garcés Ferrer, J., Administración social: servicios de bienestar social, Siglo XXI, Madrid, 1995.

AleXAnDER E.R., "Sharing Power Among Organizations", en J.M. Bryson y R. C. Einsweiler (comps.), Shared Power, University Press of America, Durham, 1991, pp. $215-250$.

AleXANDER E.R., "A Transaction Cost Theory of Planning", en Journal of the American Planning Association, 1992a, vol. 58, pp. 190-200.

AleXander E.R., Approaches to Planning, Gordon \& Breach, NuevaYork, $1992 b$

AleXANDER E.R., "To Plan or not to Plan, that is the Question: Transaction Cost Theory an Its Implication for Planning", en Environment and Planning B, 1994, vol. 21, pp.341-352.

AnsofF I., "Strategic Issue Management", en Strategic Management Journal, 1980, vol. 1, pp. 131-148. 1991.

Benvenist G., Mastering the politics of Planning, Jossey-Bass, San Francisco,

Braker J., "The Historical Concept of the Strategic Management Concept", en Academy of Management Review, 1980, vol. 5, pp. 219-224.

Bryson J.M. y Einsweiler R.C. (comps.), Strategic Planning, Planner Press, Chicago, 1988.

Bryson, J.M. y EInsweiler R.C. (comps.), Shared Powers, University Press of America, Durham, 1991.

BRYSON, J.M. y RING P.S., "A Transaction-based Approach to Policy Intervention”, en Policy Sciences, 1990, vol. 23, pp. 205.229.

Ceckoway B., (comps.), Stategic Perspective on Plannnig Practice, Lexington Books, Lexington, 1986.

Center for Philadelphia Studies, Philadelphia Investment Portfolio, University of Pennsylvania, Filadelfia, 1982.

Dahrendorf R., Markt und Plan, Mohr/Siebek, Tubinga, 1966.

DEAR M., "Understanding and Overcoming the NIMBY Syndrome", en Journal of the American Planning Association, 1992, vol. 58, pp. 288-300.

Donati P., (comp.), Fondamenti di Politica Sociale, Nis, Roma, 1993, 2 voll.

Eddison, A., Local Government:Management and Corporate Planning, Hill, Londres, 1975.

FelDMan M.S., Order Without Design: Information Production and Policy Making, Stanford University Press, Stanford, 1989.

FISHER F. y J. Forester (comps.), The Argumentative Turn in Policy Analysis and Planning, Duke University Press, Durham, 1993.

Forester J., Planning in the Face of Power, University of California Press, Berkeley, 1989. 1984.

FreEman, R. E., Strategic Management: A Stakeholder Approach, Pitman, Boston,

FriedMAN J., "A Conceptual Model for the Analysis of Planning Behaviour", en Administrative Science Quarterly, 1967, vol. 12, pp. 225-252.

Friedman J., Planning in the Public Domain, Princeton University Press, Princeton, 1987.

Granovetter M., "Economic Action and Social Structure", en American Journal of Sociology, 1985, vol. 91, pp. 481-510.
Hambelton R., "Planning Systems and Policy Implementation", en Journal of Public Policy, 1983, vol. 3, pp. 397-418. 1982.

Hall P.G., Great Planning Disasters, University of California Press, Berkeley, 1248.

Hardin G., "The Tragedy of the Commons", en Science, 1968, 162, pp. 12431973.

HaYeK F.A., Law Legislation and Liberty, University of Chicago Press, Chicago,

Herrera Gómez, M., "Conocimiento sociológico y planificación social: la relación teoría-praxis”, en Gestión y Análisis de Politicas Públicas, n. 20, 2001, pp. 71-91.

Hjern, B. y Porter D., "Implementation Structures: A New Unit of Administrative Analysis”, en Organization Studies, 1981, vol. 2, pp. 211-277.

Lindblom C.E., Politics and Markets, Basic books, New York, 1977.

Lorange P., Corporate Planning: An Executive Viewpoint, Prentice-Hall, Eglewood-Cliffs, 1980.

LUKE J. S., "Managing Interconnectdness", en J.M. Bryson e R.C. Einsweiler (comps.), Shared Power, University Press of America, Durham, 1991, pp. 25-50.

Mandelbaum S. J., "The Institutional Focus of Planning Theory", en B. Checkoway (comp.), Strategic Perspectives on Planning Practice, Lexington Book, Lexington, 1986, pp. 217-230. 1985.

Mayer R. R., Policy and Program Planning, Prentice-Hall, Eglewood Cliffs,

Mitroff I. I., Stakeholders and the Organizational Mind, Jossey-Bass, San Francisco, 1983.

Moore T., "Why Allow Planners to do what they do? A Justification from Economic Theory", en Journal of the American Association of Planners, 1978, vol. 44, pp. 387-398.

Noble D., America by Design, A. Knopf, Nueva York, 1977.

OuchI, W.G., "Markets, Hierarchics and Clans", en Administrative Science Quarterly, 1980, vol. 25, pp. 129-141.

Pagano U., "Authority, Coordination and Disequilibrium: An Explanation of the Co-exitence of Markets and Firms", en Structural Change and Economic Dynamics, 1992, vol. 3, pp. 53-77.

Palumbo D., "Implementation:What We Have Learned and Still Need to Know?", en Policy Studies Review, 1987, vol. 7, pp. 91-100.

Parsons T., The Structure of Social Action, The Free Press, Nueva York, 1937. 1957.

Parsons T., y Smelser N. J., Economy and Society, The Free Press, Nueva York,

Patton C. V., "Policy Analysis with Implementation in Mind", en B. Checkoway (comp.), Strategic Perspectives on Planning Practice, Lexington Books, Lexington, 1986, pp. 105-122.

Powell W. W., y Smith-Doerr L., "Networks and Economic Life", en N.J. Smelser, R. Swedberg (comps.), The Handbook of Economic Sociology, Sage, Beverly Hills, 1994, pp. 368-402. 1980

QuinN, J. B., Strategies for Change: Logical Incrementalism, Irwin, Homewood,

RoBins J. A., "Organizational Economics: Notes on the Use of Transaction Cost Theory in the Study of Organizations", en Administrative Science Quarterly, 1987, vol. 32, pp. 68-86. 
Rochefort D.A. y CobB, R.W. (comps.), The Politics of Problem Definition, University Press of Kansas, Kansas, 1994.

Roe E., Narrative Policy Analysis, Duke University Press, Durham, 1994.

ROGERS D. L., y Whetten D. A., Interorganizational Coordination, Iowa University Press, Ames, 1982.

SeDENBERG, P. C., The Politics of Meaning, University of Arizona Press, Tucson, 1984.

SIZA R., La programmazione e le relazioni sociali, FrancoAngeli, Milán, 1994.

Schon D. A., The Reflective Practitioner, Basic Books, Nueva York, 1983.

Susskind L. E., y Ozawa C., "Mediated Negotiation in the Public Sector: The Planner as Mediator", en Journal of Planning Education and Research, 1984, vol. 4, pp. 5-16.

TAYLOR N., "Planning Theory and the Philosophy of Planning", en Urban Studies, 1980, vol. 17, pp. 159-172.

Thompson J. D., Organizations in Action, McGraw-Hill, Nueva York, 1967.

Trinidad Requena, A., "La evaluación de los servicios sociales", en C. Alemán Bracho y J. Garcés Ferrer (dirs.), Administración social: servicios de bienestar social, Siglo XXI, Madrid, 1995, pp. 503-542.
UlriCH W., Critical Heuristics of Social Planning, Wiley, Chichester, 1994.

Veld R. J., y Schaap L., Termeer C., Twist M. (comps.), Autopoiesis and Configuration Theory: New Approaches to Societal Steering, Kluwer, Dordrecht, 1991.

WhetTen D. A., y Bozeman B., "Policy Coordination and Interorganizational Relations", en J. M. Bryson y R. C. Einsweiler (comps.), Shared Powers, University Press of America, Durham, 1991, pp. 77-104.

White, H. C., Identity and Control: A Structural Theory of Social Action, Princeton University Press, Princeton, 1992.

Williamson O. E., Market and Hierarchies, The Free Press, Nueva York, 1975.

Williamson O. E., "The Economics of Organization: Origins, Evolutions and Attributes", en Journal of Economic Literature, 1981, vol. 19, pp. 1537-1568.

Williamson O. E., The Economic Institutions of Capitalism, The Free Press, Nueva York, 1985.

Williamson O. E., "Transaction Cost Economics and Organization Theory", en N. J. Smelser y R. Swedberg (comps.), The Handbook of Economic Sociology, Sage, Beverly Hills, 1994, pp. 77-107. 
